\title{
Primes and Their Connection to Certain Polyhedral Number Sequences
}

\author{
Benjamin Lee Warren \\ Department of Mathematics, Rensselaer Polytechnic Institute, Troy 12180, New York, USA \\ Correspondence should be addressed to Benjamin Lee Warren; warreb@rpi.edu
}

Received 4 April 2021; Accepted 23 June 2021; Published 9 August 2021

Academic Editor: Marco Fontana

Copyright (c) 2021 Benjamin Lee Warren. This is an open access article distributed under the Creative Commons Attribution License, which permits unrestricted use, distribution, and reproduction in any medium, provided the original work is properly cited.

A collection of results is given regarding whether a prime can be the sum or difference of two polyhedral numbers, as well as some primality restrictions on several sequences.

\section{Introduction}

The sheer amount of various polyhedral figurate number sequences [1] makes studying them all a daunting task. Fortunately, some universal questions can be asked about them, though not all the answers are known. For instance, it is due to Euler [2] that we can find all numbers that are simultaneously triangular and square, which marks the general question of which numbers exist in more than one figurate number sequence at a time (for example, 36 is both triangular and square). Another significant result is the general question suggested by Fermat, Lagrange, Waring, Gauss, and Cauchy, which is to determine the minimal number of elements required from a given figurate sequence in order to sum to all natural numbers [3]. Though these are elegant and natural questions, few answers have so far been determined.
In this paper, another question will be examined and, very fortunately, answered for these types of sequences in general, which is as follows: when might a prime occur as the difference between two elements from some chosen figurate sequence? The answer, along with some other results, is presented..

Theorem 1. Consider the tetrahedral numbers [4] $T_{n}=(1 / 6) n(n+1)(n+2)$. Then, the only primes which are the sum of two tetrahedral numbers are $2=T_{1}+T_{1}, 5=T_{1}+T_{2}$, and $11=T_{1}+T_{3}$.

Proof. Using the closed form of $T_{n}$ along with the factorization $n(n+1)(n+2)+m(m+1) \quad(m+2)=(n+m+2)$ $\left(n^{2}+m^{2}+n+m-n m\right)$ gives the identities

$$
\begin{aligned}
T_{6 n}+T_{6 m} & =2(3 n+3 m+1)\left(6 n^{2}+6 m^{2}+n+m-6 n m\right), \\
T_{6 n}+T_{6 m+1} & =(2 n+2 m+1)\left(18 n^{2}+18 m^{2}-18 n m+9 m+1\right), \\
T_{6 n}+T_{6 m+2} & =2(3 n+3 m+2)\left(6 n^{2}+6 m^{2}-6 n m-n+5 m+1\right), \\
T_{6 n}+T_{6 m+3} & =(6 n+6 m+5)\left(6 n^{2}+6 m^{2}-6 n m-2 n+7 m+2\right), \\
T_{6 n}+T_{6 m+4} & =2(n+m+1)\left(18 n^{2}+18 m^{2}-18 n m-9 n+27 m+10\right),
\end{aligned}
$$




$$
\begin{aligned}
& T_{6 n}+T_{6 m+5}=(6 n+6 m+7)\left(6 n^{2}+6 m^{2}-6 n m-4 n+11 m+5\right), \\
& T_{6 n+1}+T_{6 m+1}=(3 n+3 m+2)\left(12 n^{2}+12 m^{2}-12 n m+4 n+4 m+1\right), \\
& T_{6 n+1}+T_{6 m+2}=(6 n+6 m+5)\left(6 n^{2}+6 m^{2}-6 n m+n+4 m+1\right), \\
& T_{6 n+1}+T_{6 m+3}=(n+m+1)\left(36 n^{2}+36 m^{2}-36 n m+36 m+11\right), \\
& T_{6 n+1}+T_{6 m+4}=(6 n+6 m+7)\left(6 n^{2}+6 m^{2}-6 n m-n+8 m+3\right), \\
& T_{6 n+1}+T_{6 m+5}=(3 n+3 m+4)\left(12 n^{2}+12 m^{2}-12 n m-4 n+20 m+9\right), \\
& T_{6 n+2}+T_{6 m+2}=2(n+m+1)\left(18 n^{2}+18 m^{2}-18 n m+9 n+9 m+4\right), \\
& T_{6 n+2}+T_{6 m+3}=(6 n+6 m+7)\left(6 n^{2}+6 m^{2}-6 n m+2 n+5 m+2\right), \\
& T_{6 n+2}+T_{6 m+4}=2(3 n+3 m+4)\left(6 n^{2}+6 m^{2}-6 n m+n+7 m+3\right), \\
& T_{6 n+2}+T_{6 m+5}=(2 n+2 m+3)\left(18 n^{2}+18 m^{2}-18 n m+27 m+13\right), \\
& T_{6 n+3}+T_{6 m+3}=(3 n+3 m+4)\left(12 n^{2}+12 m^{2}-12 n m+8 n+8 m+5\right), \\
& T_{6 n+3}+T_{6 m+4}=(2 n+2 m+3)\left(18 n^{2}+18 m^{2}-18 n m+9 n+18 m+10\right), \\
& T_{6 n+3}+T_{6 m+5}=(3 n+3 m+5)\left(12 n^{2}+12 m^{2}-12 n m+4 n+16 m+9\right), \\
& T_{6 n+4}+T_{6 m+4}=2(3 n+3 m+5)\left(6 n^{2}+6 m^{2}-6 n m+5 n+5 m+4\right), \\
& T_{6 n+4}+T_{6 m+5}=(6 n+6 m+11)\left(6 n^{2}+6 m^{2}-6 n m+4 n+7 m+5\right), \\
& T_{6 n+5}+T_{6 m+5}=(n+m+2)\left(36 n^{2}+36 m^{2}-36 n m+36 n+36 m+35\right) .
\end{aligned}
$$

Theorem 2. Consider the pyramidal numbers $P_{n}=\sum_{k=1}^{n} k^{2}=(1 / 6) n(n+1)(2 n+1)$. Then, the only primes which are the sum of two positive pyramidal numbers are $2=P_{1}+P_{1}, 19=P_{2}+P_{3}$, and $31=P_{1}+P_{4}$.
Proof. Using the closed form of $P_{n}$ along with the factorization $n(n+1)(2 n+1)+m(m+1)(2 m+1)=(n+m+$ 1) $\left(2 n^{2}+2 m^{2}-2 n m+n+m\right)$ gives the identities

$$
\begin{aligned}
P_{6 n}+P_{6 m} & =(6 n+6 m+1)\left(12 n^{2}+12 m^{2}-12 n m+n+m\right), \\
P_{6 n}+P_{6 m+1} & =(3 n+3 m+1)\left(24 n^{2}+24 m^{2}-24 n m-2 n+10 m+1\right), \\
P_{6 n}+P_{6 m+2} & =(2 n+2 m+1)\left(36 n^{2}+36 m^{2}-36 n m-9 n+27 m+5\right), \\
P_{6 n}+P_{6 m+3} & =(3 n+3 m+2)\left(24 n^{2}+24 m^{2}-24 n m-10 n+26 m+7\right), \\
P_{6 n}+P_{6 m+4} & =(6 n+6 m+5)\left(12 n^{2}+12 m^{2}-12 n m-7 n+17 m+6\right), \\
P_{6 n}+P_{6 m+5} & =(n+m+1)\left(72 n^{2}+72 m^{2}-72 n m-54 n+126 m+55\right), \\
P_{6 n+1}+P_{6 m+1} & =(2 n+2 m+1)\left(36 n^{2}+36 m^{2}-36 n m+9 n+9 m+2\right), \\
P_{6 n+1}+P_{6 m+2} & =(3 n+3 m+2)\left(24 n^{2}+24 m^{2}-24 n m+2 n+14 m+3\right), \\
P_{6 n+1}+P_{6 m+3} & =(6 n+6 m+5)\left(12 n^{2}+12 m^{2}-12 n m-n+11 m+3\right), \\
P_{6 n+1}+P_{6 m+4} & =(n+m+1)\left(72 n^{2}+72 m^{2}-72 n m-18 n+90 m+31\right), \\
P_{6 n+1}+P_{6 m+5} & =(6 n+6 m+7)\left(12 n^{2}+12 m^{2}-12 n m-5 n+19 m+8\right), \\
P_{6 n+2}+P_{6 m+2} & =(6 n+6 m+5)\left(12 n^{2}+12 m^{2}-12 n m+5 n+5 m+2\right), \\
P_{6 n+2}+P_{6 m+3} & =(n+m+1)\left(72 n^{2}+72 m^{2}-72 n m+18 n+54 m+19\right), \\
P_{6 n+2}+P_{6 m+4}= & (6 n+6 m+7)\left(12 n^{2}+12 m^{2}-12 n m+n+13 m+5\right), \\
P_{6 n+2}+P_{6 m+5} & =(3 n+3 m+4)\left(24 n^{2}+24 m^{2}-24 n m-2 n+34 m+15\right), \\
P_{6 n+3}+P_{6 m+3} & =(6 n+6 m+7)\left(12 n^{2}+12 m^{2}-12 n m+7 n+7 m+4\right),
\end{aligned}
$$




$$
\begin{aligned}
& P_{6 n+3}+P_{6 m+4}=(3 n+3 m+4)\left(24 n^{2}+24 m^{2}-24 n m+10 n+22 m+11\right), \\
& P_{6 n+3}+P_{6 m+5}=(2 n+2 m+3)\left(36 n^{2}+36 m^{2}-36 n m+9 n+45 m+23\right), \\
& P_{6 n+4}+P_{6 m+4}=(2 n+2 m+3)\left(36 n^{2}+36 m^{2}-36 n m+27 n+27 m+20\right), \\
& P_{6 n+4}+P_{6 m+5}=(3 n+3 m+5)\left(24 n^{2}+24 m^{2}-24 n m+14 n+26 m+17\right), \\
& P_{6 n+5}+P_{6 m+5}=(6 n+6 m+11)\left(12 n^{2}+12 m^{2}-12 n m+11 n+11 m+10\right) .
\end{aligned}
$$

Lemma 1. Consider the pyramidal numbers $P_{n}=(1 / 6) n(n+1)(2 n+1)$. Then, for all $n \in \mathbb{N}$, the following identity follows by elementary algebra:

$$
P_{n(2 n+3)}+P_{(n+1)(2 n-1)}=2 P_{2 n(n+1)} \text {. }
$$

Theorem 3. Consider the octahedral numbers $O_{n}=(1 / 3) n\left(2 n^{2}+1\right)$. Then, the only primes which are the sum of two positive octahedral numbers are $2=O_{1}+O_{1}$ and $7=\mathrm{O}_{1}+\mathrm{O}_{2}$.

Proof. Using the closed form of $O_{n}$ along with the factorization $n\left(2 n^{2}+1\right)+m\left(2 m^{2}+1\right)=(n+m)\left(2 n^{2}+2 m^{2}-\right.$ $2 n m+1)$ gives the identities

$$
\begin{aligned}
O_{3 n}+O_{3 m} & =(n+m)\left(18 n^{2}+18 m^{2}-18 n m+1\right), \\
O_{3 n}+O_{3 m+1} & =(3 n+3 m+1)\left(6 n^{2}+6 m^{2}-6 n m-2 n+4 m+1\right), \\
O_{3 n}+O_{3 m+2} & =(3 n+3 m+2)\left(6 n^{2}+6 m^{2}-6 n m-4 n+8 m+3\right), \\
O_{3 n+1}+O_{3 m+1} & =(3 n+3 m+2)\left(6 n^{2}+6 m^{2}-6 n m+2 n+2 m+1\right), \\
O_{3 n+1}+O_{3 m+2} & =(n+m+1)\left(18 n^{2}+18 m^{2}-18 n m+18 m+7\right), \\
O_{3 n+2}+O_{3 m+2} & =(3 n+3 m+4)\left(6 n^{2}+6 m^{2}-6 n m+4 n+4 m+3\right) .
\end{aligned}
$$

Theorem 4. Consider the dodecahedral numbers $\mathscr{D}_{n}=(1 / 2) n(3 n-1)(3 n-2)$. Then, the only prime which is the sum of two dodecahedral numbers is $2=\mathscr{D}_{1}+\mathscr{D}_{1}$.
Proof. Using the closed form of $\mathscr{D}_{n}$ along with the factorization $n(3 n-1)(3 n-2)+m(3 m-1)(3 m-2)=(3 n+$ $3 m-2)\left(3 n^{2}+3 m^{2}-3 n m-n-m\right)$ gives the identities

$$
\begin{aligned}
\mathscr{D}_{2 n}+\mathscr{D}_{2 m} & =2(3 n+3 m-1)\left(6 n^{2}+6 m^{2}-6 n m-n-m\right), \\
\mathscr{D}_{2 n}+\mathscr{D}_{2 m+1} & =(6 n+6 m+1)\left(6 n^{2}+6 m^{2}-6 n m-4 n+5 m+1\right), \\
\mathscr{D}_{2 n+1}+\mathscr{D}_{2 m+1} & =(3 n+3 m+2)\left(12 n^{2}+12 m^{2}-12 n m+4 n+4 m+1\right) .
\end{aligned}
$$

Theorem 5. Consider the icosahedral numbers $I_{n}=(1 / 2)$ $n\left(5 n^{2}-5 n+2\right)$. Then, primes which are the difference between two icosahedral numbers $p=I_{a}-I_{b}$ occur only if $a-b \leq 2$.
Proof. Using the closed form of $I_{n}$ along with the factorization $n\left(5 n^{2}-5 n+2\right)-m\left(5 m^{2}-5 m+2\right)=(n-m)\left(5 n^{2}\right.$ $\left.+5 m^{2}+5 n m-5 n-5 m+2\right)$ gives the identities 


$$
\begin{aligned}
I_{2 n}-I_{2 m} & =2(n-m)\left(10 n^{2}+10 m^{2}+10 n m-5 n-5 m+1\right), \\
I_{2 n}-I_{2 m+1} & =(2 n-2 m-1)\left(10 n^{2}+10 m^{2}+10 n m+5 m+1\right), \\
I_{2 n+1}-I_{2 m+1} & =(n-m)\left(20 n^{2}+20 m^{2}+20 n m+20 n+20 m+7\right) .
\end{aligned}
$$

The first several primes which are the difference between two icosahedral numbers are $11=I_{2}-I_{1}, 47=I_{3}-I_{1}$, $131=I_{5}-I_{4}$, etc.

Theorem 6. Consider the truncated tetrahedral numbers $\tau_{n}=(1 / 6) n\left(23 n^{2}-27 n+10\right)$. Then, primes which are the difference between two truncated tetrahedral numbers $p=$ $\tau_{a}-\tau_{b}$ occur only if $a-b \leq 6$.

Proof. Using the closed form of $\tau_{n}$ along with the factorization $n\left(23 n^{2}-27 n+10\right)-m\left(23 m^{2}-27 m+10\right)=(n-$ m) $\left(23 n^{2}+23 m^{2}+23 n m-27 n-27 m+10\right)$ gives the identities

$$
\begin{aligned}
& \tau_{6 n}-\tau_{6 m}=2(n-m)\left(414 n^{2}+414 m^{2}+414 n m-81 n-81 m+5\right), \\
& \tau_{6 n}-\tau_{6 m+1}=(6 n-6 m-1)\left(138 n^{2}+138 m^{2}+138 n m-4 n+19 m+1\right), \\
& \tau_{6 n}-\tau_{6 m+2}=(6 n-6 m-2)\left(138 n^{2}+138 m^{2}+138 n m+19 n+65 m+8\right), \\
& \tau_{6 n}-\tau_{6 m+3}=(2 n-2 m-1)\left(414 n^{2}+414 m^{2}+414 n m+126 n+333 m+68\right) \text {, } \\
& \tau_{6 n}-\tau_{6 m+4}=2(3 n-3 m-2)\left(138 n^{2}+138 m^{2}+138 n m+65 n+157 m+45\right) \text {, } \\
& \tau_{6 n}-\tau_{6 m+5}=(6 n-6 m-5)\left(138 n^{2}+138 m^{2}+138 n m+88 n+203 m+75\right), \\
& \tau_{6 n+1}-\tau_{6 m+1}=(n-m)\left(828 n^{2}+828 m^{2}+828 n m+252 n+252 m+25\right), \\
& \tau_{6 n+1}-\tau_{6 m+2}=(6 n-6 m-1)\left(138 n^{2}+138 m^{2}+138 n m+65 n+88 m+15\right) \text {, } \\
& \tau_{6 n+1}-\tau_{6 m+3}=(3 n-3 m-1)\left(276 n^{2}+276 m^{2}+276 n m+176 n+268 m+67\right) \text {, } \\
& \tau_{6 n+1}-\tau_{6 m+4}=(2 n-2 m-1)\left(414 n^{2}+414 m^{2}+414 n m+333 n+540 m+179\right), \\
& \tau_{6 n+1}-\tau_{6 m+5}=(3 n-3 m-2)\left(276 n^{2}+276 m^{2}+276 n m+268 n+452 m+187\right), \\
& \tau_{6 n+2}-\tau_{6 m+2}=2(n-m)\left(414 n^{2}+414 m^{2}+414 n m+333 n+333 m+89\right) \text {, } \\
& \tau_{6 n+2}-\tau_{6 m+3}=(6 n-6 m-1)\left(138 n^{2}+138 m^{2}+138 n m+134 n+157 m+52\right) \text {, } \\
& \tau_{6 n+2}-\tau_{6 m+4}=2(3 n-3 m-1)\left(138 n^{2}+138 m^{2}+138 n m+157 n+203 m+82\right) \text {, } \\
& \tau_{6 n+2}-\tau_{6 m+5}=(2 n-2 m-1)\left(414 n^{2}+414 m^{2}+414 n m+540 n+747 m+359\right) \text {, } \\
& \tau_{6 n+3}-\tau_{6 m+3}=(n-m)\left(828 n^{2}+828 m^{2}+828 n m+1080 n+1080 m+469\right), \\
& \tau_{6 n+3}-\tau_{6 m+4}=(6 n-6 m-1)\left(138 n^{2}+138 m^{2}+138 n m+203 n+226 m+112\right) \text {, } \\
& \tau_{6 n+3}-\tau_{6 m+5}=(3 n-3 m-1)\left(276 n^{2}+276 m^{2}+276 n m+452 n+544 m+307\right) \text {, } \\
& \tau_{6 n+4}-\tau_{6 m+4}=2(n-m)\left(414 n^{2}+414 m^{2}+414 n m+747 n+747 m+449\right), \\
& \tau_{6 n+4}-\tau_{6 m+5}=(6 n-6 m-1)\left(138 n^{2}+138 m^{2}+138 n m+272 n+295 m+195\right) \text {, } \\
& \tau_{6 n+5}-\tau_{6 m+5}=(n-m)\left(828 n^{2}+828 m^{2}+828 n m+1908 n+1908 m+1465\right) .
\end{aligned}
$$


The first several primes which are the difference between two truncated tetrahedral numbers are $67=\tau_{3}-\tau_{1}$, $179=\tau_{4}-\tau_{1}, 359=\tau_{5}-\tau_{2}$, etc.

Theorem 7. Consider the truncated cube numbers $K_{n}=(1 / 3)\left(77 n^{3}+69 n^{2}+19 n+3\right)$. Then, primes which are the difference between two truncated cube numbers $p=K_{a}-$ $K_{b}$ occur only if $a-b \leq 3$.

Proof. Using the closed form of $K_{n}$ along with the factorization $\quad 77 n^{3}+69 n^{2}+19 n-77 m^{3}-69 m^{2}-19 m=(n-$ m) $\left(77 n^{2}+77 m^{2}+77 n m+69 n+69 m+19\right)$ gives the identities

$$
\begin{aligned}
K_{3 n}-K_{3 m} & =(n-m)\left(693 n^{2}+693 m^{2}+693 n m+207 n+207 m+19\right), \\
K_{3 n}-K_{3 m+1} & =(3 n-3 m-1)\left(231 n^{2}+231 m^{2}+231 n m+146 n+223 m+55\right), \\
K_{3 n}-K_{3 m+2} & =(3 n-3 m-2)\left(231 n^{2}+231 m^{2}+231 n m+223 n+377 m+155\right), \\
K_{3 n+1}-K_{3 m+1} & =(n-m)\left(693 n^{2}+693 m^{2}+693 n m+900 n+900 m+388\right), \\
K_{3 n+1}-K_{3 m+2} & =(3 n-3 m-1)\left(231 n^{2}+231 m^{2}+231 n m+377 n+454 m+255\right), \\
K_{3 n+2}-K_{3 m+2} & =(n-m)\left(693 n^{2}+693 m^{2}+693 n m+1593 n+1593 m+1219\right) .
\end{aligned}
$$

The first several primes which are the difference between two truncated cube numbers are $919=K_{4}-K_{1}$, $1117=K_{5}-K_{4}, 14221=K_{10}-K_{7}$, etc.

Theorem 8. Consider the triakis tetrahedral numbers $\pi_{n}=(1 / 2) n\left(3 n^{2}-3 n+2\right)$. Then, $\pi_{a}-\pi_{b}$ is prime only if $a-b \leq 2$.
Proof. Using $\pi_{n}=(1 / 2) n\left(3 n^{2}-3 n+2\right)$ along with the factorization $n\left(3 n^{2}-3 n+2\right)-m\left(3 m^{2}-3 m+2\right)=(n-$ m) $\left(3 n^{2}+3 m^{2}+3 n m-3 n-3 m+2\right)$ gives the identities

$$
\begin{aligned}
\pi_{2 n}-\pi_{2 m} & =2(n-m)\left(6 n^{2}+6 m^{2}+6 n m-3 n-3 m+1\right), \\
\pi_{2 n}-\pi_{2 m+1} & =(2 n-2 m-1)\left(6 n^{2}+6 m^{2}+6 n m+3 m+1\right) \\
\pi_{2 n+1}-\pi_{2 m+1} & =(n-m)\left(12 n^{2}+12 m^{2}+12 n m+12 n+12 m+5\right) .
\end{aligned}
$$

The first several primes which are the difference between two triakis tetrahedral numbers are $7=\pi_{2}-\pi_{1}$, $29=\pi_{3}-\pi_{1}, 79=\pi_{5}-\pi_{4}$, etc.
Proof. Using $\sigma_{n}=(1 / 2) n\left(7 n^{2}-9 n+4\right)$ along with the factorization $n\left(7 n^{2}-9 n+4\right)-m\left(7 m^{2}-9 m+4\right)=(n-m)$ $\left(7 n^{2}+7 m^{2}+7 n m-9 n-9 m+4\right)$ gives the identities

Theorem 9. Consider the triakis octahedral numbers $\sigma_{n}=(1 / 2) n\left(7 n^{2}-9 n+4\right)$. Then, $\sigma_{a}-\sigma_{b}$ is prime only if $a-b \leq 2$.

$$
\begin{aligned}
\sigma_{2 n}-\sigma_{2 m} & =2(n-m)\left(14 n^{2}+14 m^{2}+14 n m-9 n-9 m+2\right), \\
\sigma_{2 n}-\sigma_{2 m+1} & =(2 n-2 m-1)\left(14 n^{2}+14 m^{2}+14 n m-2 n+5 m+1\right), \\
\sigma_{2 n+1}-\sigma_{2 m+1} & =(n-m)\left(28 n^{2}+28 m^{2}+28 n m+24 n+24 m+7\right) .
\end{aligned}
$$

The first several primes which are the difference between two triakis octahedral numbers are $13=\sigma_{2}-\sigma_{1}$, $59=\sigma_{3}-\sigma_{1}, 271=\sigma_{5}-\sigma_{4}$, etc.
Theorem 10. Consider the tetrakis hexahedral numbers $\theta_{n}=(1 / 3) n\left(10 n^{2}-12 n+5\right)$. Then, $\theta_{a}-\theta_{b}$ is prime only if $a-b \leq 3$. 
Proof. Using $\theta_{n}=(1 / 3) n\left(10 n^{2}-12 n+5\right)$ along with the factorization $n\left(10 n^{2}-12 n+5\right)-m\left(10 m^{2}-12 m+5\right)=$ $(n-m)\left(10 n^{2}+10 m^{2}+10 n m-12 n-12 m+5\right)$ gives the identities

$$
\begin{aligned}
\theta_{3 n}-\theta_{3 m} & =(n-m)\left(90 n^{2}+90 m^{2}+90 n m-36 n-36 m+5\right), \\
\theta_{3 n}-\theta_{3 m+1} & =(3 n-3 m-1)\left(30 n^{2}+30 m^{2}+30 n m-2 n+8 m+1\right), \\
\theta_{3 n}-\theta_{3 m+2} & =(3 n-3 m-2)\left(30 n^{2}+30 m^{2}+30 n m+8 n+28 m+7\right), \\
\theta_{3 n+1}-\theta_{3 m+1} & =(n-m)\left(90 n^{2}+90 m^{2}+90 n m+54 n+54 m+11\right), \\
\theta_{3 n+1}-\theta_{3 m+2} & =(3 n-3 m-1)\left(30 n^{2}+30 m^{2}+30 n m+28 n+38 m+13\right), \\
\theta_{3 n+2}-\theta_{3 m+2} & =(n-m)\left(90 n^{2}+90 m^{2}+90 n m+144 n+144 m+77\right) .
\end{aligned}
$$

The first several primes which are the difference between two tetrakis hexahedral numbers are $13=\theta_{2}-\theta_{1}$, $97=\theta_{4}-\theta_{3}, 311=\theta_{5}-\theta_{2}$, etc.

Theorem 11. Consider the triangular numbers $\Delta_{n}=(1 / 2) n(n+1)$ with $n \in \mathbb{N}$. Then, primes which are the difference between two triangular numbers $p=\Delta_{a}-\Delta_{b}$ occur only if $a-b \leq 2$.

Proof. Using $\Delta_{n}=(1 / 2) n(n+1)$ along with the identity $n(n+1)-m(m+1)=(n-m)(n+m+1)$ gives the following three equations:

$$
\begin{aligned}
\Delta_{2 n}-\Delta_{2 m} & =(n-m)(2 n+2 m+1), \\
\Delta_{2 n}-\Delta_{2 m+1} & =(n+m+1)(2 n-2 m-1), \\
\Delta_{2 n+1}-\Delta_{2 m+1} & =(n-m)(2 n+2 m+3) .
\end{aligned}
$$

The first several primes which are the difference between two triangular numbers are $2=\Delta_{2}-\Delta_{1}, \quad 5=\Delta_{3}-\Delta_{1}$, $7=\Delta_{4}-\Delta_{2}$, etc.

Corollary 1. $\sum_{n=b}^{a} n$ is prime only if $a-b=1$.

Proof. Using the well-known result $\sum_{m=0}^{n} m=$ $(1 / 2) n(n+1)=\Delta_{n}$, it follows that $\sum_{n=b}^{a} n=\Delta_{a}-\Delta_{b-1}$, so by the previous theorem, a prime of the form $p=\sum_{n=b}^{a} n$ exists only if $a-(b-1) \leq 2 \Rightarrow a-b=1$.

Theorem 12. Consider the triakis icosahedral numbers $\gamma_{n}=(1 / 2) n\left(19 n^{2}-27 n+10\right)$. Then, $\gamma_{a}-\gamma_{b}$ is prime only if $a-b \leq 2$.

Proof. Using $\gamma_{n}=(1 / 2) n\left(19 n^{2}-27 n+10\right)$ along with the factorization $n\left(19 n^{2}-27 n+10\right)-m\left(19 m^{2}-27 m+10\right)$ $=(n-m)\left(19 n^{2}+19 m^{2}+19 n m-27 n-27 m+10\right)$ gives the identities

$$
\begin{aligned}
\gamma_{2 n}-\gamma_{2 m} & =2(n-m)\left(38 n^{2}+38 m^{2}+38 n m-27 n-27 m+5\right), \\
\gamma_{2 n}-\gamma_{2 m+1} & =(2 n-2 m-1)\left(38 n^{2}+38 m^{2}+38 n m-8 n+11 m+1\right), \\
\gamma_{2 n+1}-\gamma_{2 m+1} & =(n-m)\left(76 n^{2}+76 m^{2}+76 n m+60 n+60 m+13\right) .
\end{aligned}
$$

The first several primes which are the difference between two triakis icosahedral numbers are $31=\gamma_{2}-\gamma_{1}$, $149=\gamma_{3}-\gamma_{1}, 463=\gamma_{5}-\gamma_{4}$, etc.
Theorem 13. Consider the pentakis dodecahedral numbers $\rho_{n}=(1 / 6) n\left(55 n^{2}-75 n+26\right)$. Then, $\rho_{a}-\rho_{b}$ is prime only if $a-b \leq 6$. 
Proof. Using $\rho_{n}=(1 / 6) n\left(55 n^{2}-75 n+26\right)$ along with the factorization $n\left(55 n^{2}-75 n+26\right)-m\left(55 m^{2}-75 m+26\right)$ $=(n-m)\left(55 n^{2}+55 m^{2}+55 n m-75 n-75 m+26\right)$ gives the identities

$$
\begin{aligned}
\rho_{6 n}-\rho_{6 m} & =2(n-m)\left(990 n^{2}+990 m^{2}+990 n m-225 n-225 m+13\right), \\
\rho_{6 n}-\rho_{6 m+1} & =(6 n-6 m-1)\left(330 n^{2}+330 m^{2}+330 n m-20 n+35 m+1\right), \\
\rho_{6 n}-\rho_{6 m+2} & =2(3 n-3 m-1)\left(330 n^{2}+330 m^{2}+330 n m+35 n+145 m+16\right), \\
\rho_{6 n}-\rho_{6 m+3} & =(2 n-2 m-1)\left(990 n^{2}+990 m^{2}+990 n m+270 n+765 m+148\right), \\
\rho_{6 n}-\rho_{6 m+4} & =2(3 n-3 m-2)\left(330 n^{2}+330 m^{2}+330 n m+145 n+365 m+101\right), \\
\rho_{6 n}-\rho_{6 m+5} & =(6 n-6 m-5)\left(330 n^{2}+330 m^{2}+330 n m+200 n+475 m+171\right), \\
\rho_{6 n+1}-\rho_{6 m+1} & =(n-m)\left(1980 n^{2}+1980 m^{2}+1980 n m+540 n+540 m+41\right), \\
\rho_{6 n+1}-\rho_{6 m+2} & =(6 n-6 m-1)\left(330 n^{2}+330 m^{2}+330 n m+145 n+200 m+31\right), \\
\rho_{6 n+1}-\rho_{6 m+3} & =(3 n-3 m-1)\left(660 n^{2}+660 m^{2}+660 n m+400 n+620 m+147\right), \\
\rho_{6 n+1}-\rho_{6 m+4} & =(2 n-2 m-1)\left(990 n^{2}+990 m^{2}+990 n m+765 n+1260 m+403\right), \\
\rho_{6 n+1}-\rho_{6 m+5} & =(3 n-3 m-2)\left(660 n^{2}+660 m^{2}+660 n m+620 n+1060 m+427\right), \\
\rho_{6 n+2}-\rho_{6 m+2} & =2(n-m)\left(990 n^{2}+990 m^{2}+990 n m+765 n+765 m+193\right), \\
\rho_{6 n+2}-\rho_{6 m+3} & =(6 n-6 m-1)\left(330 n^{2}+330 m^{2}+330 n m+310 n+365 m+116\right), \\
\rho_{6 n+2}-\rho_{6 m+4} & =2(3 n-3 m-1)\left(330 n^{2}+330 m^{2}+330 n m+365 n+475 m+186\right), \\
\rho_{6 n+2}-\rho_{6 m+5} & =(2 n-2 m-1)\left(990 n^{2}+990 m^{2}+990 n m+1260 n+1755 m+823\right), \\
\rho_{6 n+3}-\rho_{6 m+3}=(n-m)\left(1980 n^{2}+1980 m^{2}+1980 n m+2520 n+2520 m+1061\right), & \\
\rho_{6 n+3}-\rho_{6 m+4}= & (6 n-6 m-1)\left(330 n^{2}+330 m^{2}+330 n m+475 n+530 m+256\right), \\
\rho_{6 n+4}-\rho_{6 m+4}=2(n-m)\left(990 n^{2}+990 m^{2}+990 n m+1755 n+1755 m+1033\right), & =(3 n-3 m-1)\left(660 n^{2}+660 m^{2}+660 n m+1060 n+1280 m+707\right), \\
\rho_{6 n+5}-\rho_{6 m+5}= & (n-m)\left(1980 n^{2}+1980 m^{2}+1980 n m+4500 n+4500 m+3401\right),
\end{aligned}
$$

The first several primes which are the difference between two pentakis dodecahedral numbers are $31=\rho_{2}-\rho_{1}$, $701=\rho_{6}-\rho_{5}, 823=\rho_{5}-\rho_{2}$, etc.

Theorem 14. Consider the disdyakis triacontahedral numbers $\Lambda_{n}=(1 / 3) n\left(55 n^{2}-75 n+23\right)$. Then, $\Lambda_{a}-\Lambda_{b}$ is prime only if $a-b \leq 3$.
Proof. Using $\Lambda_{n}=(1 / 3) n\left(55 n^{2}-75 n+23\right)$ along with the factorization $n\left(55 n^{2}-75 n+23\right)-m\left(55 m^{2}-75 m+23\right)$ $=(n-m)\left(55 n^{2}+55 m^{2}+55 n m-75 n-75 m+23\right)$ gives the identities

$$
\begin{aligned}
\Lambda_{3 n}-\Lambda_{3 m} & =(n-m)\left(495 n^{2}+495 m^{2}+495 n m-225 n-225 m+23\right), \\
\Lambda_{3 n}-\Lambda_{3 m+1} & =(3 n-3 m-1)\left(165 n^{2}+165 m^{2}+165 n m-20 n+35 m+1\right), \\
\Lambda_{3 n}-\Lambda_{3 m+2} & =(3 n-3 m-2)\left(165 n^{2}+165 m^{2}+165 n m+35 n+145 m+31\right), \\
\Lambda_{3 n+1}-\Lambda_{3 m+1} & =(n-m)\left(495 n^{2}+495 m^{2}+495 n m+270 n+270 m+38\right), \\
\Lambda_{3 n+1}-\Lambda_{3 m+2} & =(3 n-3 m-1)\left(165 n^{2}+165 m^{2}+165 n m+145 n+200 m+61\right), \\
\Lambda_{3 n+2}-\Lambda_{3 m+2} & =(n-m)\left(495 n^{2}+495 m^{2}+495 n m+765 n+765 m+383\right) .
\end{aligned}
$$


The first several primes which are the difference between two disdyakis triacontahedral numbers are $61=\Lambda_{2}-\Lambda_{1}$, $2011=\Lambda_{7}-\Lambda_{6}, 6143=\Lambda_{8}-\Lambda_{5}$, etc.

Theorem 15. Consider the pentagonal numbers $p_{n}=(1 / 2) n(3 n-1)$. Then, $p_{a}-p_{b}$ is prime only if $a-b \leq 2$.

Proof

$$
\begin{aligned}
p_{2 n}-p_{2 m} & =(n-m)(6 n+6 m-1), \\
p_{2 n}-p_{2 m+1} & =(2 n-2 m-1)(3 n+3 m+1), \\
p_{2 n+1}-p_{2 m+1} & =(n-m)(6 n+6 m+5) .
\end{aligned}
$$

The first several primes which are the difference between two pentagonal numbers are $7=p_{3}-p_{2}, 11-p_{3}-p_{1}$, $11-p_{3}-p_{1} 17=p_{4}-p_{2}$, etc.

There is a universal generalization of some of these which is encompassed in the following several results.

Lemma 2. Let $a_{0}, a_{1}, \ldots, a_{R} \in \mathbb{R}$. Then, there exists the following algebraic identity:

$$
n \sum_{k=0}^{R} a_{k} n^{k}-m \sum_{k=0}^{R} a_{k} m^{k}=(n-m) \sum_{j=0}^{R} n^{j} \sum_{\ell=j}^{R} a_{\ell} m^{\ell-j} .
$$

Now, the universal result is ready to be given.

Theorem 16. Let $\left\{A_{n}\right\}_{n=0}^{\infty}$ be a sequence of the form $A_{n}=$ $(1 / z)\left(g+n \sum_{k=0}^{R} a_{k} n^{k}\right)$ satisfying the following:

(1) $z, g \in \mathbb{Z}$ and $a_{i} \in \mathbb{Z}$ for all $i=0: R$

(2) $z$ is the largest positive integer that divides $n \sum_{k=0}^{R} a_{k} n^{k}$ for all positive integers $n$

(3) $A_{n}$ is a positive integer for all $n \in \mathbb{N}$

Then, $A_{a}-A_{b}$ is prime only if $a-b \leq z$.

Proof. Let $x, y \in \mathbb{N}$ such that $0 \leq x<z$ and $0 \leq y<z$. Then, letting $a=z n+x$ and $b=z m+y$ in Lemma 2 gives $A_{a}-A_{b}=A_{z n+x}-A_{z m+y}=(1 / z) U V$, where $U=z n-z m+$ $x-y$ and $V=\sum_{j=0}^{R}(z n+x)^{j} \sum_{\ell=j}^{R} a_{\ell}(z m+y)^{\ell-j}$. Now, there will be three scenarios.

Scenario 1. $x=y$ and $n>m$. If this is true, then $z$ divides $U$ as $U / z=n-m$ which when set equal to 1 gives $n=m+1$ which means $a-b=z$.

Scenario 2. $x>y$ and $n \geq m$. If this is true, there will be three cases:

Case 1: some of the factors of $z$ divide $U$ and some of the factors of $z$ divide $V$ but not all the factors of $z$ divide $U$ or $V$. This is impossible since it would imply that $A_{a}-A_{b}$ is not an integer, but it clearly is an integer.
Case 2: $z$ divides $U$ or $z$ divides $V$. If $z$ divides $U$, then we have Scenario 1 . If $z$ divides $V$, then the only values of $n$ and $m$ which can make $V / z$ equal to 1 correspond to $n=m=0$ which indicates that $a-b=x-y<z$.

Case 3: some of the factors of $z$ divide $U$ and the rest of the factors of $z$ divide $V$. If this is true, then $U$ will reduce into $(z / \operatorname{gcd}(z, x-y))(n-m)+$ $(x-y / \operatorname{gcd}(z, x-y))$ which if set equal to 1 gives $n=$ $m+(1 / z)(\operatorname{gcd}(z, x-y)+y-x)$. These are integers only if $(1 / z)(\operatorname{gcd}(z, x-y)+y-x)$ is an integer, and this can occur only if $\operatorname{gcd}(x, x-y)+y-x=0$. This indicates that $n=m$, so $a-b=x-y<z$. Also, when $V$ is reduced, then it can be 1 only if $n=m=0$, so $a-b=x-y<z$.

Scenario 3. $x<y$ and $n>m$. If this is true, then there will be the same three cases as in Scenario 2.

Case 1: this case was shown to be impossible.

Case 2: this case is similar to case 2 in Scenario 2.

Case 3: $U$ will reduce into $(z / \operatorname{gcd}(z, y-x))(n-m)+$ $(x-y / \operatorname{gcd}(z, y-x))$ which if set equal to 1 gives $n=m+(1 / z)(\operatorname{gcd}(z, x-y)+y-x)$. These are integers only if $(1 / z)(\operatorname{gcd}(z, y-x)+y-x)$ is an integer, and this can occur only if $\operatorname{gcd}(x, y-x)+y-x=1$. This indicates that $n=m+1$, so $a-b=z+x-y<z$. Also, when $V$ is reduced, then it can be equal to 1 only if $n=m=0$, but this will not happen since $n>m$.

All the polyhedra in this paper have associated polyhedral number sequences that are of the form referred to by the previous theorem. Many more $R$-dimensional polyhedra not discussed here also have associated sequences that are of the type in Theorem 15.

Many statements follow as an immediate result from Theorem 15, such as the statement $\left(\begin{array}{l}n \\ k\end{array}\right)-\left(\begin{array}{c}m \\ k\end{array}\right)$ is prime only if $n-m \leq k !$. Another one is that $\sum_{k=0}^{n} k^{p}-\sum_{k=0}^{m} k^{p}$ is prime only if $n-m \leq Q$, where $Q$ is the reduced denominator in the Faulhaber formula evaluated at a given positive integer $p$. Another statement that follows from Theorem 15 is that if $g_{n}(k)$ denotes the $n$th polygonal number from a polygon with $k$ sides $g_{n}(k)=(1 / 2) n[n k-2 n-k+4]$, then $g_{a}(k)-g_{b}(k)$ is prime only if $a-b=1$ if $k$ is even or $a-$ $b \leq 2$ if $k$ is odd. One more great statement that follows from Theorem 15 is that if $\mathscr{P}_{n}(k)$ denotes the $n$th number from a polygonal pyramid from a polygon with $k$ sides $\mathscr{P}_{n}(k)=(1 / 6) n\left(k n^{2}-2 n^{2}+3 n-k+5\right), \quad$ then $\mathscr{P}_{a}(k)$ $-\mathscr{P}_{b}(k)$ is prime only if $a-b \leq 6 / \operatorname{gcd}(3, k-2,|5-k|)$.

There is also another result for a sequence which is a case of the sequences referred to in Theorem 15, but it has another very striking property, as shown in the following theorem. 
Theorem 17. Let $\left\{B_{n}\right\}_{n=0}^{\infty}$ be a sequence of the form $B_{n}=$ $(1 / z) n \sum_{k=0}^{R} a_{k} n^{k}$ satisfying

(1) $z \in \mathbb{Z}$ and $a_{i} \in \mathbb{Z}$ for all $i=0: R$

(2) $z$ divides $n \sum_{k=0}^{R} a_{k} n^{k}$ for all positive integers $n$

(3) $B_{-n}=-B_{n}$ for all $n \in \mathbb{Z}$

(4) $B_{n}$ is a positive integer for all $n \in \mathbb{N}$

Then, for each $R \in \mathbb{N}$, there are at most finitely many primes of the form $p=B_{a}+B_{b}$.

Proof. Replace $m$ with $-m$ in Lemma 2, and apply the assumption that every exponent in $n \sum_{k=0}^{R} a_{k} n^{k}$ is odd.

This will give the identity $n \sum_{k=0}^{R}$ $a_{k} n^{k}+m \sum_{k=0}^{R} a_{k} m^{k}=(n+m) \sum_{j=0}^{R} n^{j} \sum_{\ell=j}^{R} a_{\ell}(-1)^{\ell-j} m^{\ell-j}$.

So, now define positive integers $x, y$ as $0 \leq x<z$ and $0 \leq y<z$, and let $a=z n+x$ and $b=z m+y$. Then, $B_{a}+B_{b}=B_{z n+x}+B_{z m+y}=(1 / z) \mu \nu$, where $\mu=z n+z m+x$ $+y$ and $v=\sum_{j=0}^{R}(z n+x)^{j} \sum_{\ell=j}^{R} a_{\ell}(-1)^{\ell-j}(z m+y)^{\ell-j}$. So, now there will be three cases:
Case 1: $z$ divides $\mu$ or $z$ divides $\nu$. If this is true, $B_{a}+B_{b}$ will factor into two positive polynomials either of which will be 1 only if $n=m=0$, so there will be at most finitely many primes of the form $p=B_{a}+B_{b}$.

Case 2: some of the factors of $z$ divide $\mu$ and the rest of the factors of $z$ divide $v$. If this is true, then like case 1 , $B_{a}+B_{b}$ will factor into two positive polynomials, either of them can be 1 only if $n=m=0$, so there will be at most finitely many primes of the form $p=B_{a}+B_{b}$.

Case 3: some of the factors of $z$ divide $\mu$ and some of the factors of $z$ divide $\nu$ but not all the factors of $z$ divide $\mu$ or $\nu$. This case is impossible because it would imply that $B_{a}+B_{b}$ is not an integer, but it clearly is an integer.

Several more results include some other interesting properties of some polyhedral numbers.

The following identity can be verified with elementary algebra.

Lemma 3

$$
a(a+b)(a+c)+d(c+d-b)(c+d)=(a+c+d)\left(a^{2}+d^{2}+a b+c d-a d-b d\right)
$$

Using some cases of this lemma leads to the following theorems.

Theorem 18. Let $C(n, r, k)=(1 / k) n(n+r)(n+2 r)$ for positive integers $n, r$, and $k$. Then, for all fixed $r$ and $k$, there are at most finitely many primes of the form $p=C(n, r, k)+C(m, r, k)$.

Proof. First, note that from Lemma 3, it follows that $n(n+$ r) $(n+2 r)+m(m+r)$

$(m+2 r)=(n+m+2 r)\left(n^{2}+m^{2}+2 r m+n r-n m-r m\right)$.

Now, for two positive integers $a$ and $b$, let $n=k n^{\prime}+a$ and $m=k m^{\prime}+b$.

Then, $C(n, r, k)+C(m, r, k)=C\left(k n^{\prime}+a, r, k\right)+C(k$ $\left.m^{\prime}+b, r, k\right)=(1 / k) Y Z$, where $Y=k n^{\prime}+k m^{\prime}+a+b+2 r$ and $Z=a^{2}+b^{2}+a r+b r-a b+k^{2} n^{\prime 2}+k^{2} m^{\prime 2}+k n^{\prime} r+k m^{\prime} r$ $+2 k n^{\prime} a+2 k m^{\prime} b-k^{2} n^{\prime} m^{\prime}-k n^{\prime} b-k m^{\prime} a$. Now, there will be two cases:

Case 1: $k$ divides $a+b+2 r$ or $k$ divides $a^{2}+b^{2}+a r+b r-a b$. Then, from this, $k$ divides either $Y$ or $Z$, so $C(n, r, k)+C(m, r, k)$ runs through at most finitely many primes.

Case 2: $k$ does not divide $a+b+2 r$ and $k$ does not divide $a^{2}+b^{2}+a r+b r-a b$.

In this case, there are three scenarios.

Scenario 4. If there are no similar factors between $k$ and $a+$ $b+2 r$ and there are no similar factors between $k$ and $a^{2}+b^{2}+a r+b r-a b$, then $k$ is coprime to both $Y$ and $Z$ which means $A(n, r, k)+A(m, r, k)$ is not an integer.

Scenario 5. If there are some similar factors between $k$ and $a+b+2 r$ and the rest of the factors of $k$ are similar to some factors of $a^{2}+b^{2}+a r+b r-a b$, then ( $\left.1 / k\right) Y Z$ would simplify into the product of two polynomial factors, which means $C(n, r, k)+C(m, r, k)$ runs through at most finitely many primes.

Scenario 6. If there are some similar factors between $k$ and $a+b+2 r$ or some similar factors between $k$ and $a^{2}+b^{2}+$ $a r+b r-a b$ but not all the factors of $k$ are similar to the factors of both $a+b+2 r$ and $a^{2}+b^{2}+a r+b r-a b$, then $(1 / k) Y Z$ will reduce into $\left(1 / k^{\prime}\right) Y^{\prime} Z^{\prime}$, but $k^{\prime}$ is coprime to both $Y^{\prime}$ and $Z^{\prime}$ which means $C(n, r, k)+C(m, r, k)$ is not an integer.

From this, it follows that if $C(n, r, k)+C(m, r, k)$ is an integer for some fixed $r$ and $k$, it must factor into two positive polynomials, so there can only be finitely many primes of the form $p=C(n, r, k)+C(m, r, k)$.

Corollary 2. Consider the numbers $F_{n}=(1 / 4) n(n+1)(n+$ 2) for $n \in \mathbb{N}$. Then, the only prime of the form $p=F_{n}+F_{m}$ is $3=F_{1}+F_{1}$.

Proof. Letting $r=1$ and $k=4$ in Theorem 17 gives the identities 


$$
\begin{aligned}
F_{4 n}+F_{4 m} & =2(2 n+2 m+1)\left(4 n^{2}+4 m^{2}-4 n m+n+m\right), \\
F_{4 n}+F_{4 m+1} & =\frac{1}{2}(4 n+4 m+3)\left(8 n^{2}+8 m^{2}-8 n m+6 m+1\right), \\
F_{4 n}+F_{4 m+2} & =(n+m+1)\left(16 n^{2}+16 m^{2}-16 n m-4 n+20 m+6\right), \\
F_{4 n}+F_{4 m+3} & =(4 n+4 m+5)\left(4 n^{2}+4 m^{2}-4 n m-2 n+7 m+3\right), \\
F_{4 n+1}+F_{4 m+1} & =(n+m+1)\left(16 n^{2}+16 m^{2}-16 n m+8 n+8 m+3\right), \\
F_{4 n+1}+F_{4 m+2} & =\frac{1}{2}(4 n+4 m+5)\left(8 n^{2}+8 m^{2}-8 n m+2 n+8 m+3\right), \\
F_{4 n+1}+F_{4 m+3} & =\frac{1}{2}(2 n+2 m+3)\left(16 n^{2}+16 m^{2}-16 n m+24 m+11\right), \\
F_{4 n+2}+F_{4 m+2} & =2(2 n+2 m+3)\left(4 n^{2}+4 m^{2}-4 n m+3 n+3 m+2\right), \\
F_{4 n+2}+F_{4 m+3} & =(4 n+4 m+7)\left(4 n^{2}+4 m^{2}-4 n m+2 n+5 m+3\right), \\
F_{4 n+3}+F_{4 m+3} & =(n+m+2)\left(16 n^{2}+16 m^{2}-16 n m+16 n+16 m+15\right) .
\end{aligned}
$$

Theorem 19. Consider the triangular numbers $\Delta_{n}=n(n+1) / 2$, and let $n, m$, and $k$ be nonnegative integers. Then, the only primes that are of the form $p=(n+k+1) \Delta_{n}+m \Delta_{m+k}$ are $2=(1+0+1) \Delta_{1}+0 \Delta_{0+0}$ and $3=(1+1+1) \Delta_{1}+0 \Delta_{0+1}=(1+0+1) \Delta_{1}+1 \Delta_{1+0}=$ $(0+1+1) \Delta_{0}+1 \Delta_{2}$.
Proof. Let $G(n, m, k)=(n+k+1) \Delta_{n}+m \Delta_{m+k}$. Using $\Delta_{n}=$ $n(n+1) / 2$ and the fact that $n(n+1)(n+k+1)+m(m+$ k) $(m+k+1)=(n+m+k+1)\left(n^{2}+m^{2}-n m+n+k m\right)$, it is easy to verify the following identities:

$$
\begin{aligned}
G(2 n, 2 m, 2 k) & =(2 n+2 m+2 k+1)\left(2 n^{2}+2 m^{2}-2 n m+2 m k+n\right), \\
G(2 n, 2 m, 2 k+1) & =2(n+m+k+1)\left(2 n^{2}+2 m^{2}-2 n m+2 m k+n+m\right), \\
G(2 n, 2 m+1,2 k) & =(n+m+k+1)\left(4 n^{2}+4 m^{2}-4 n m+4 m k+4 m+2 k+1\right), \\
G(2 n, 2 m+1,2 k+1) & =(2 n+2 m+2 k+3)\left(2 n^{2}+2 m^{2}-2 n m+2 m k+3 m+k+1\right), \\
G(2 n+1,2 m, 2 k) & =2(n+m+k+1)\left(2 n^{2}+2 m^{2}-2 n m+2 m k+3 n-m+1\right), \\
G(2 n+1,2 m, 2 k+1) & =(2 n+2 m+2 k+3)\left(2 n^{2}+2 m^{2}-2 n m+2 m k+3 n+1\right), \\
G(2 n+1,2 m+1,2 k) & =(2 n+2 m+2 k+3)\left(2 n^{2}+2 m^{2}-2 n m+2 m k+2 n+m+k+1\right), \\
G(2 n+1,2 m+1,2 k+1) & =(n+m+k+2)\left(4 n^{2}+4 m^{2}-4 n m+4 m k+4 n+4 m+2 k+3\right) .
\end{aligned}
$$

Lemma 4. Consider the tetrahedral numbers $T_{n}=(1 / 6) n(n+1)(n+2)$. Then, for all $n \in \mathbb{N}$, the following identity can be shown with elementary algebra:

$$
T_{n(n+3)}+T_{(n+1)(n+4)}=2 T_{(n+1)(n+3)} .
$$

Theorem 20. Still using $T_{n}=(1 / 6) n(n+1)(n+2)$, there exists the identity

$$
\sum_{m=1}^{n}(-1)^{n+m} T_{m(m+2)}=\frac{1}{2} T_{n(n+3)} .
$$


Proof. From $T_{n(n+3)}+T_{(n-1)(n+2)}=2 T_{n(n+2)}$, we can assemble the list of equations:

$$
\begin{aligned}
T_{n(n+3)}+T_{(n-1)(n+2)} & =2 T_{n(n+2)}, \\
-T_{(n-1)(n+2)}-T_{(n-2)(n+1)} & =-2 T_{(n-1)(n+1)}, \\
T_{(n-2)(n+1)}+T_{(n-3) n} & =2 T_{(n-2) n}, \\
-T_{(n-3) n}-T_{(n-4)(n-1)} & =-2 T_{(n-3)(n-1)}, \\
(-1)^{n+1} T_{(1)(4)} & =2(-1)^{n+1} T_{(1)(3)} .
\end{aligned}
$$
$T_{n(n+3)}$.

Adding up all these gives $2 \sum_{m=1}^{n}(-1)^{n+m} T_{m(m+2)}=$

\section{Conclusion}

We now have a result to determine very easily whether the difference between two elements from a polyhedral number sequence may be eligible to be a prime. We also see that the only platonic number sequence [5] in which it is unknown whether there are infinitely primes as the sum of two elements is the icosahedral number.

There exist some additional following identities relating some of these sequences to each other. Let $\Delta_{n}=(1 / 2) n(n+$ 1) denote the $n$th triangular number, $T_{n}=(1 / 6) n(n+1)$ $(n+2)$ denote the $n$th tetrahedral number, $O_{n}=(1 / 3) n\left(2 n^{2}+1\right)$ denote the $n$th octahedral number, and $P_{n}=(1 / 6) n(n+1)(2 n+1)$ denote the $n$th pyramidal number.

\section{Lemma 5}

$$
\left(\begin{array}{c}
2 n+1 \\
4
\end{array}\right)+(n+1)^{4}=\left(\begin{array}{c}
2 n+4 \\
4
\end{array}\right)+n^{4} .
$$

\section{Lemma 6}

$$
T_{n+2}+P_{n+1}=T_{n}+P_{n+2}
$$

Lemma 7

$$
T_{n^{2}-2}+n^{4}=T_{n^{2}}
$$

\section{Lemma 8}

$$
T_{(n+2)^{2}}+O_{\Delta_{n}}=T_{(n+1)^{2}}+O_{\Delta_{n+2}}
$$

\section{Data Availability}

The mathematical data used to support the findings of this study are included within the article.

\section{Conflicts of Interest}

The author declares that there are no conflicts of interest.

\section{Acknowledgments}

All funding invested in the article is provided by the author.

\section{References}

[1] E. Deza and M. Deza, Figurate Numbers, vol. 93, World Scientific Publishing, Singapore, 2012.

[2] L. Euler, "Regula facilis problemata Diophantea per numeros integros expedite resolvendi (an easy rule for Diophantine problems which are to be resolved quickly by integral numbers)," Memoires de l'Academie des Sciences de St.-Petersbourg, vol. 4, no. 3, p. 17, 1813.

[3] Nathanson and B. Melvyn, Additive Number Theory The Classical Bases, Springer, vol. 164, Berlin, Germany, 1996.

[4] W. Sierpinski, "On tetrahedral numbers (in Polish)," pp. 209-217, 1964.

[5] T. Pappas, The Five Platonic Solids, The Joy of Mathematics, pp. 110-111, Wide World Publishing, San Carlos, CA, USA, 1989. 